\title{
A New Radar Scan Mode to Increase Clear-Air Velocity Data Coverage and Usability for Wind Analysis
}

\author{
QIN XU \\ NOAA/OAR/National Severe Storms Laboratory, Norman, Oklahoma \\ KANG NAI AND VALERY MELNIKOV \\ Cooperative Institute for Mesoscale Meteorological Studies, University of Oklahoma, and NOAA/OAR/National \\ Severe Storms Laboratory, Norman, Oklahoma
}

(Manuscript received 14 November 2018, in final form 11 April 2019)

\begin{abstract}
By using long pulses with extended dwell time, radar sensitivity is enhanced such that more clear-air returns are usable. This enhanced capability can increase clear-air data coverage and provide additional information for radar wind analysis and data assimilation. To explore this potential capability and related benefits, a new scan mode with long pulses and low antenna rotation rate is derived from the existing clear-air scan mode and applied to the named KOUN S-band test bed radar at NSSL. Velocities collected using this newly modified scan mode are significantly enhanced in data coverage compared to those collected with the unmodified scan mode from the nearby test bed radar (named KCRI). However, transmitter duty cycle limits the pulse repetition frequency (PRF) to $455 \mathrm{~Hz}$ for the long pulses, which reduces the Nyquist velocity to $12.53 \mathrm{~m} \mathrm{~s}^{-1}$ for the new scan mode. Such a small Nyquist interval leads to significantly worse problems with aliased velocities. A recently developed dealiasing algorithm is modified by using external reference velocities from KCRI to overcome increased difficulties in dealiasing, making the velocities from KOUN useful over increased range and area. This implies that similar increases in velocity data coverage can be achieved without using external reference velocities if the newly modified scan is paired with an unmodified scan into dual-PRF scans. This dual-PRF approach is proposed for future development.
\end{abstract}

\section{Introduction}

Since the network of Weather Surveillance Radar1988 Doppler (WSR-88D) systems [known as Next Generation Weather Radar (NEXRAD)] was deployed and installed to modernize the U.S. weather radar systems, the NEXRAD has played an important role in improving short-range forecasts and warnings for severe thunderstorms, tornadoes, and flash floods (Crum et al. 1998; Whiton et al. 1998). In particular, the increased receiver sensitivity and real-time display provided by the WSR-88D systems and automated algorithms subsequently developed have enabled National Weather Service (NWS) forecasters to detect severe storms and related threatening wind conditions and to provide warnings with improved accuracy and lead time (Stumpf et al. 1998; Simmons and Sutter 2005; Lakshmanan et al. 2007; Brotzge and Donner 2013). The precipitation

Corresponding author: Dr. Qin Xu, qin.xu@noaa.gov products provided by the systems have been used for hydrologic and natural resource applications, especially in high-intensity events that greatly affect soil erosion and flooding (Hardegree et al. 2008). These accomplished the major milestones originally projected for the NEXRAD program (Crum and Alberty 1993). In addition, beyond the originally projected major milestones for the NEXRAD program, high-resolution reflectivity and velocity observations have been ingested into the operational data assimilation systems at the National Centers for Environmental Prediction (NCEP) to improve operational numerical weather predictions (Liu et al. 2016).

However, as the utilization of NEXRAD data by the three operating agencies (i.e., the NWS, Department of Defense, and Federal Aviation Administration) is primarily for the detection and modeling of extreme weather events, NEXRAD data collected under clear-air weather conditions have not found much usage due to shallow and limited radial-range 
coverage $(\leq 50 \mathrm{~km})$ in clear air and the relatively large spacing between radars $(\approx 300 \mathrm{~km})$. Except for detecting sea-breeze fronts and outflow boundaries, most of the clear-air return is not used by forecasters (Crum et al. 1998). Yet, reflectivity from clear-air Bragg scatter (caused by temperature and moisture irregularities having scales of half the radar wavelength) have been used to detect changes in surface fluxes of sensible and latent heats (Rabin and Doviak 1989) and to estimate the height of the convective boundary layer (Heinselman et al. 2009; Elmore et al. 2012; Melnikov et al. 2011, 2013; Richardson et al. 2017). Convective boundary layer heights may be used as "proxy observations" in data assimilation, but the original clear-air reflectivities are often not purely meteorological (mainly due to the presence of insects, as well as bats and birds) and are difficult to assimilate directly. On the other hand, clear-air Doppler velocity observations are mostly meteorological as they are not or not much affected by the presence of insects, while contaminated velocity observations caused by the presence of bats and birds, especially migrating birds, can be largely detected and removed through data quality control (Zhang et al. 2005; Liu et al. 2005; Jiang et al. 2013). After adequate data quality control, the clear-air velocity observations can be directly assimilated to improve numerical weather predictions especially if their spatial coverage can be enhanced. Motivated by this, the current study aims to examine weather and how the existing scan modes used for clear-air by the WSR-88Ds can be modified to enhance the detectability and thus increase the coverage of velocity observations.

To explore this and related benefits, a new scan mode with long pulses and a slow scan rate is designed by modifying the existing scan mode for the research and development (R\&D) S-band radar (named KOUN) at NSSL. This new scan mode is detailed in the next section with brief descriptions of problems in velocity dealiasing and required modifications to the previously developed dealiasing techniques (Xu and Nai 2012; Xu et al. 2013). Raw velocities collected from KOUN using the new scan mode and dealiased velocities produced successfully by using a stand-alone version of dealiasing (modified from the previously developed dealiasing) are presented in section 3 in comparison with those from the nearby test bed radar (named KCRI) using the unmodified scan mode. Raw velocities collected from KOUN using the new scan mode are often very difficult to dealias even by using the aforementioned stand-alone dealiasing, but when combined with KCRI add what amounts to a dual-PRF approach and mitigate difficulties in KOUN dealiasing. This approach is presented in section 4, with conclusions in section 5.

\section{New scan mode and velocity dealiasing}

The WSR-88Ds are capable of transmitting long $(4.5 \mu \mathrm{s})$ and short $(1.5 \mu \mathrm{s})$ pulses. Due to transmitter duty cycle limitations, the maximum PRFs at the long and short pulses are 455 and $1280 \mathrm{~Hz}$, respectively. The long pulse mode results in more sensitivity than the short pulse mode, but at the cost of a smaller Nyquist velocity, denoted by $V_{N}$. In clear-air situations, the WSR-88Ds operate in two volume coverage patterns (VCPs): VCP31 uses long pulses while VCP32 uses short pulses with a high pulse count to improve detectability. To cover the maximum specified range of $460 \mathrm{~km}$ (truncated from $471 \mathrm{~km}$ ) in the contiguous surveillance scan of VCP31, the KOUN radar operates at a PRF of $318 \mathrm{~Hz}$, while the contiguous Doppler scan of VCP31 is operated at a PRF of $435 \mathrm{~Hz}$ with a Nyquist velocity of $V_{N}=12 \mathrm{~m} \mathrm{~s}^{-1}$. To maximize the Nyquist velocity in the long pulse mode, a new experimental VCP with a PRF of $455 \mathrm{~Hz}$ is designed for KOUN. The Nyquist velocity at this VCP is improved to $12.53 \mathrm{~m} \mathrm{~s}^{-1}$, which is used with our dealiasing algorithm, described below. Because of a reduced azimuthal scan rate, the new VCP has a longer dwell time than VCP31 and VCP32. In particular, the new VCP has three contiguous Doppler (CD) velocity scans at $0.5^{\circ}, 1.5^{\circ}$, and $2.5^{\circ}$ in each volume scan and completes the entire volume scan in $10.5 \mathrm{~min}$, while the VCP31 or VCP32 has five paired contiguous surveillance (CS) reflectivity and CD velocity scans at $0.5^{\circ}, 1.5^{\circ}$, $2.5^{\circ}, 3.5^{\circ}$, and $4.5^{\circ}$ in each volume scan and completes the entire volume scan in $9 \mathrm{~min} 50 \mathrm{~s}$. The volume update time $(10.5 \mathrm{~min})$ of the new VCP is slightly longer than that (9 min $50 \mathrm{~s})$ of VCP31 and VCP32 but still sufficiently rapid to resolve the temporal variations of wind fields on the mesoscale, especially in clear-air situations. For the new VCP, the choice of three elevations at $0.5^{\circ}$, $1.5^{\circ}$, and $2.5^{\circ}$ in each volume scan is due partially to the constrained update time (about $10 \mathrm{~min}$ ) and partially to the fact that radar velocity observations at elevations higher than $5^{\circ}$ are less useful and thus not used in operational radar data assimilation at NCEP (according to S. Liu 2019, personal communication).

Detailed specifications of VCP31 and VCP32 versus the new VCP at the same three lowest elevations $\left(0.5^{\circ}\right.$, $1.5^{\circ}$, and $2.5^{\circ}$ ) are listed in Table 1 . As shown by the last column of Table 1, for the new VCP at each elevation, the unambiguous range, $R_{\mathrm{um}}$, is reduced slightly from that of the VCP31 CD scan but is much larger than that of the VCP32 CD scan. Since $R_{\mathrm{um}}>300 \mathrm{~km}$ for the new VCP, range folding is unlikely in clear air, but might 
TABLE 1. Specifications of VCP31 and VCP32 vs new VCP at the same three lowest elevations $\left(0.5^{\circ}, 1.5^{\circ}\right.$, and $\left.2.5^{\circ}\right)$. Here, $\mathrm{CS}$ and CD stand for contiguous surveillance and contiguous Doppler, respectively, and $R_{\mathrm{um}}$ denotes the unambiguous range.

\begin{tabular}{|c|c|c|c|c|c|c|c|c|c|c|}
\hline $\begin{array}{l}\text { Sweep } \\
\text { No. }\end{array}$ & $\begin{array}{l}\text { Elevation } \\
\quad\left({ }^{\circ}\right)\end{array}$ & $\begin{array}{l}\text { VCP } \\
\text { No. }\end{array}$ & $\begin{array}{l}\text { Pulse } \\
\text { length }\end{array}$ & $\begin{array}{l}\text { PRF } \\
(\mathrm{Hz})\end{array}$ & $\begin{array}{c}\text { No. of } \\
\text { samples }\end{array}$ & $\begin{array}{l}\text { Azimuthal rate } \\
\qquad\left({ }^{\circ} \mathrm{s}^{-1}\right)\end{array}$ & $\begin{array}{l}\text { Dwell time } \\
\text { (s) }\end{array}$ & $\begin{array}{c}V_{N} \\
\left(\mathrm{~m} \mathrm{~s}^{-1}\right)\end{array}$ & Type & $\begin{array}{r}R_{\mathrm{um}} \\
(\mathrm{km})\end{array}$ \\
\hline \multirow[t]{2}{*}{1} & 0.5 & 31 & Long & 318 & 63 & 5.04 & 71 & - & $\mathrm{CS}$ & 471 \\
\hline & & 32 & Short & 318 & 63 & 5.04 & 71 & - & $\mathrm{CS}$ & 471 \\
\hline \multirow[t]{3}{*}{2} & 0.5 & 31 & Long & 435 & 87 & 5.07 & 72 & 12.0 & $\mathrm{CD}$ & 344 \\
\hline & & 32 & Short & 1021 & 224 & 4.57 & 79 & 28.2 & $\mathrm{CD}$ & 146 \\
\hline & & New & Long & 455 & 265 & 1.72 & 210 & 12.5 & CD & 329 \\
\hline \multirow[t]{2}{*}{3} & 1.5 & 31 & Long & 318 & 63 & 5.04 & 71 & - & $\mathrm{CS}$ & 471 \\
\hline & & 32 & Short & 318 & 63 & 5.04 & 71 & - & $\mathrm{CS}$ & 471 \\
\hline \multirow[t]{3}{*}{4} & 1.5 & 31 & Long & 435 & 87 & 5.07 & 72 & 12.0 & $\mathrm{CD}$ & 344 \\
\hline & & 32 & Short & 1021 & 224 & 4.57 & 79 & 28.2 & $\mathrm{CD}$ & 146 \\
\hline & & New & Long & 455 & 265 & 1.72 & 210 & 12.5 & $\mathrm{CD}$ & 329 \\
\hline \multirow[t]{2}{*}{5} & 2.5 & 31 & Long & 318 & 63 & 5.04 & 71 & - & CS & 471 \\
\hline & & 32 & Short & 435 & 87 & 5.07 & 72 & - & CS & 344 \\
\hline \multirow[t]{3}{*}{6} & 2.5 & 31 & Long & 435 & 87 & 5.07 & 72 & 12.0 & $\mathrm{CD}$ & 344 \\
\hline & & 32 & Short & 1021 & 224 & 4.57 & 79 & 28.2 & $\mathrm{CD}$ & 146 \\
\hline & & New & Long & 455 & 265 & 1.72 & 210 & 12.5 & $\mathrm{CD}$ & 329 \\
\hline
\end{tabular}

occur at the lowest elevation $\left(0.5^{\circ}\right)$ if the new VCP is also used (beyond its intended clear-air applications) to improve detectability of weak echoes in winter precipitation or some mixed precipitation events, and this could be a potential limitation of the new VCP. However, since no range folding is seen from the KOUN velocity data collected in this study using the new VCP even under rainy weather conditions, we will leave the concerned potential limitation and possible remedy for future studies.

Using this new VCP scan mode, Doppler velocities were collected from KOUN for the time period from 16 to 20 April in 2016. During this period, the atmospheric condition changed from clear-air to cloudy and rainy, and followed by scattered precipitation and intermittent clear-air conditions, as each convective rainband initiated along the east side of a prefrontal dryline and propagated westward traversing the KOUN radar site. However, since the PRF was limited to $455 \mathrm{~Hz}$ leading to a Nyquist velocity of $V_{N}=12.53 \mathrm{~m} \mathrm{~s}^{-1}$, the collected (level II) raw velocities were severely aliased (as shown in Figs. $1 \mathrm{a}-\mathrm{c}$ ) and created enormous difficulties in dealiasing.

Note that the aforementioned VCP31 and VCP32 for clear-air situations are also often used for winter precipitation events. The long-pulse VCP31 (with $V_{N}$ reduced below $12 \mathrm{~m} \mathrm{~s}^{-1}$ ) is susceptible to velocity dealiasing errors, and raw velocities scanned with VCP31 from severe winter ice storms can be severely aliased (often with the aliased velocities folded twice in high-wind areas above the inversion layers). The two-dimensional velocity dealiasing algorithm (2DVDA) of Jing and Wiener (1993) is the default velocity dealiasing scheme operationally used on the WSR-88D. This 2DVDA was optimized and tested for broad operational applications (Witt et al. 2009). As the algorithm must have considerable tolerance for bad or poor-quality data to retain as much as possible the original data coverage (to satisfy the primary need for visual applications at the weather forecast offices), the processed velocity data are not free of serious aliasing errors especially for velocities scanned with VCP31 [see Tables 1-3 of Witt et al. (2009)], and thus cannot satisfy the high-quality standard required by operational data assimilation at NCEP.

To meet the need of radar data assimilation, persistent efforts have been made at NSSL to develop robust dealiasing techniques (Gong et al. 2003; Gao et al. 2004; Xu et al. 2011; Xu and Nai 2012; Xu et al. 2013). In particular, an alias-robust variational analysis (AR-Var) was developed (Xu and Nai 2013) by using the unconventional approach (Xu et al. 2009) with the aliasing operator derived in terms of Bayesian estimation (Xu 2009) to mimic the effect of aliasing that causes discontinuities in radial velocity observations in the cost function. This AR-Var can be applied directly to aliased raw velocities to produce reliable reference radial velocities for the reference check in the AR-Var-based dealiasing free of aliasing errors even for velocities scanned with VCP31 [as shown in Table 2 of Xu and Nai (2012)]. The AR-Var-based dealiasing was extended adaptively for various different VCPs and further improved by using the hourly predicted wind fields from the NCEP operational Rapid Refresh model as the first guess for the reference check via a model-assisted approach [see section 3 of Xu et al. (2013)]. This modelassisted approach is implemented in the latest version of AR-Var-based dealiasing, and it can substantially increase the seed data coverage produced by the reference 

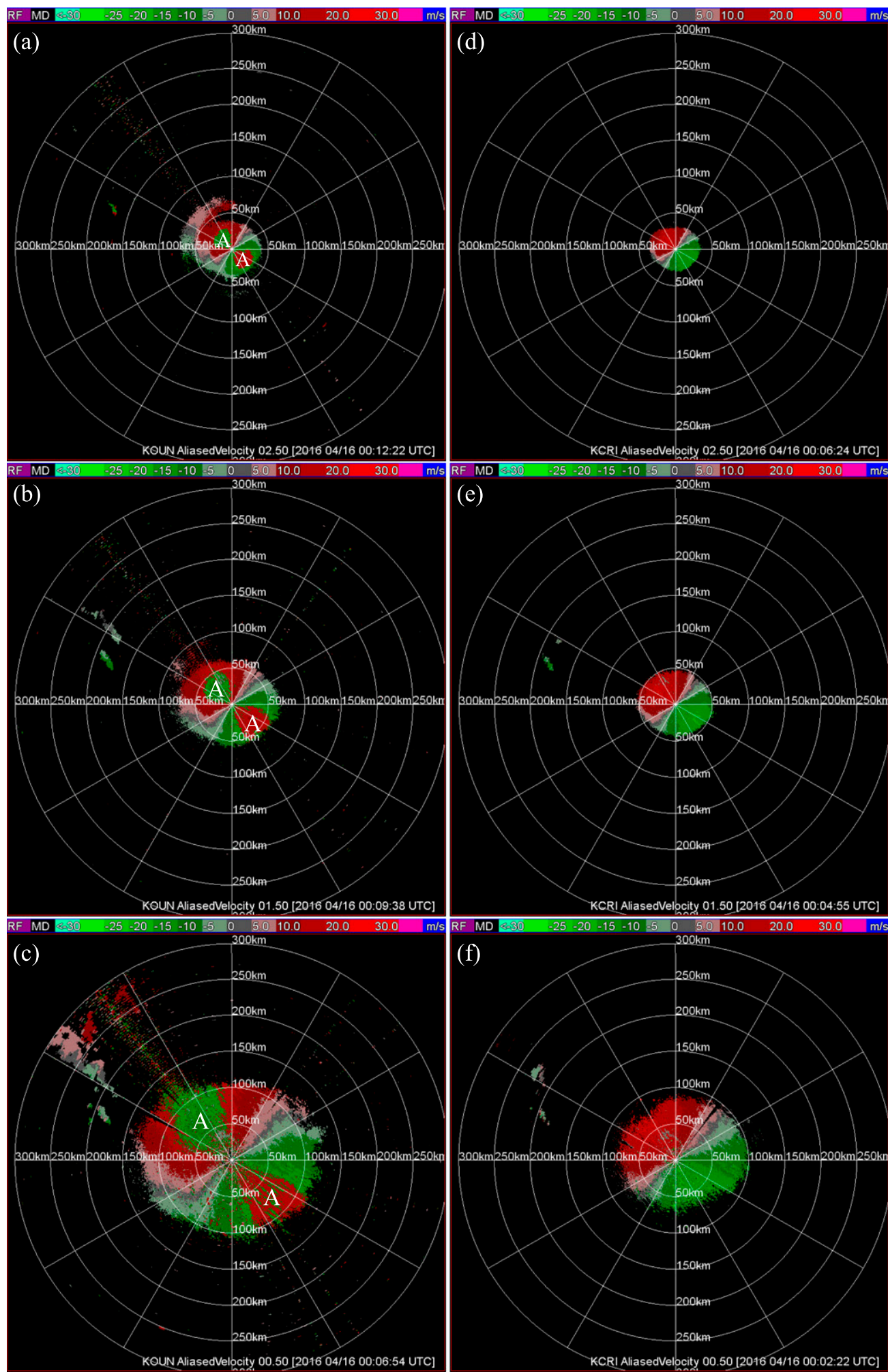

FIG. 1. (a)-(c) Images of raw (level-II) velocities on the three sweeps scanned at $2.5^{\circ}, 1.5^{\circ}$, and $0.5^{\circ}$ from KOUN using the newly modified scan mode (with the Nyquist velocity reduced to $12.53 \mathrm{~m} \mathrm{~s}^{-1}$ ) under a clearair weather condition around 0000 UTC 16 Apr 2016. (d)-(f) As in (a)-(c), but scanned from KCRI using the existing unmodified scan mode (with a Nyquist velocity of $25 \mathrm{~m} \mathrm{~s}^{-1}$ ). The white "A"s in (a)-(c) mark the aliased-velocity areas. 
check in the first step and thus increase the final dealiased data coverage [see Table 1 of Xu et al. (2013)]. However, limited by the accuracies of current numerical model predictions, such a model-assisted approach is not yet suitable or adequate to overcome the aforementioned difficulties in dealiasing severely aliased raw velocities collected from KOUN with the new VCP scan mode, so the above reviewed dealiasing technique must be modified adaptively to overcome the successively increased difficulties in dealiasing.

First, the dealiasing technique is modified back into a stand-alone version without using reference velocities from numerical model predictions for the reference check performed in the first step [see section 3 of $\mathrm{Xu}$ et al. (2013)], because the accuracy required for each reference velocity [that must be within $\pm 1.2 V_{N}$ of the true radial velocity, as explained in section 2 of $\mathrm{Xu}$ and Nai (2012)] becomes more stringent (due to the reduced $V_{N}$ ) than the current numerical model predictions can achieve. In this stand-alone version, reference velocities are produced by applying the AR-Var to raw velocities scanned from KOUN using the new scan mode. In this case, the reference check in the first step is similar to that developed previously by Xu and Nai (2012) for winter ice storms scanned by VCP31 where the Nyquist velocity is reduced to $12 \mathrm{~m} \mathrm{~s}^{-1}$ or less. However, the continuity check in the second step of dealiasing is no longer limited by the radial range covered by the seed data produced by the reference check in the first step [see section 2 of $\mathrm{Xu}$ and Nai (2012)]. This stand-alone AR-Var-based dealiasing, called SA-VDA for short, can be applied to raw velocities from KOUN using the new scan mode (with $V_{N}$ reduced to $12.53 \mathrm{~m} \mathrm{~s}^{-1}$ ) to produce dealiased velocities with increased coverage for various time periods under various weather conditions, but not for all conditions over the entire period (from 16 to 20 April).

Often, raw velocities from KOUN using the new scan mode are too noisy near the radar and/or too severely aliased for the AR-Var analysis to produce reference velocities for the reference check. Intractable raw velocities are thus all discarded by the SA-VDA. The raw velocities from KCRI are also noisy but not as degraded as those from KOUN, and so can still be processed by the SA-VDA. Dealiased velocities from KCRI can be used as proxy references for the reference check applied to the raw velocities scanned from KOUN at about the same time and same elevation angle as those scanned from KCRI. This is the first type of modification made to the above SA-VDA and it amounts to a simulated dualPRF approach. As will be shown in section $4 \mathrm{a}$, this modification is effective as long as the SA-VDA can dealias raw velocities from KCRI.
On some occasions, the raw velocities collected from KCRI became too noisy near the radar and/or too sparse to be used by the AR-Var analysis and thus discarded by the SA-VDA, but these raw velocities were free or almost free of aliasing and thus can be used directly as proxy references for the reference check applied to the raw velocities from KOUN. This is the second type of modification made to the SA-VDA. As will be shown in section $4 \mathrm{~b}$, this modification is effective as long as the aliased velocities from KCRI are rare. Again, as proxy references, the alias-free raw velocities from KCRI are used in this paper to mimic alias-free raw velocities obtainable internally from KOUN, via a dual-PRF approach. We will revisit the above two types of dual-PRF approach in the conclusions section.

\section{Results obtained using SA-VDA}

\section{a. Results obtained under clear-air weather condition}

Raw velocities scanned from KOUN using the new scan mode in clear-air around 0000 UTC 16 April 2016 are shown by the images in Figs. 1a-c (for three sweeps at $2.5^{\circ}, 1.5^{\circ}$, and $0.5^{\circ}$ ). In comparison, raw velocities scanned from the nearby KCRI radar $(314 \mathrm{~m}$ northeast from KOUN radar site) around the same time are shown by the images in Figs. 1d-f (also for three sweeps at $2.5^{\circ}, 1.5^{\circ}$, and $0.5^{\circ}$ ). Clearly, the new scan mode can increase the data coverage in radial ranges at least $25 \%$ and up to $100 \%$ in this case. In particular, the range of coverage to the west and northwest of the radar was increased from 75 to $130 \mathrm{~km}$ at $0.5^{\circ}$, from 50 to $75 \mathrm{~km}$ at $1.5^{\circ}$, and from 30 to $60 \mathrm{~km}$ at $2.5^{\circ}$. In Figs. $1 \mathrm{a}-\mathrm{c}$, the white "A"s mark the aliased-velocity areas. In particular, the contiguous discontinuities in color for the KOUN raw velocity images in Figs. 1a-c (as opposed to the smoothly changing colors for the KCRI raw velocity images in Figs. 1d-f) reveal that the raw velocities from KOUN are aliased.

These aliased velocities are mostly detected and corrected by the SA-VDA, so the dealiased data have almost the same coverage as the original raw data (see Figs. 2a-c in comparison with Figs. 1a-c). However, because the operational WSR-88D ground clutter filter has not been fine-tuned for the new long-pulse VCP (with a long dwell time) but was used when the level-I KOUN data (i.e., the recorded output of the radar digital receiver, also know as time series of in-phase and quadrature data) were converted to level-II data (i.e., digital radial base data output from a radar signal processor that include reflectivity, radial velocity and spectrum), the level-II velocity data from KOUN 

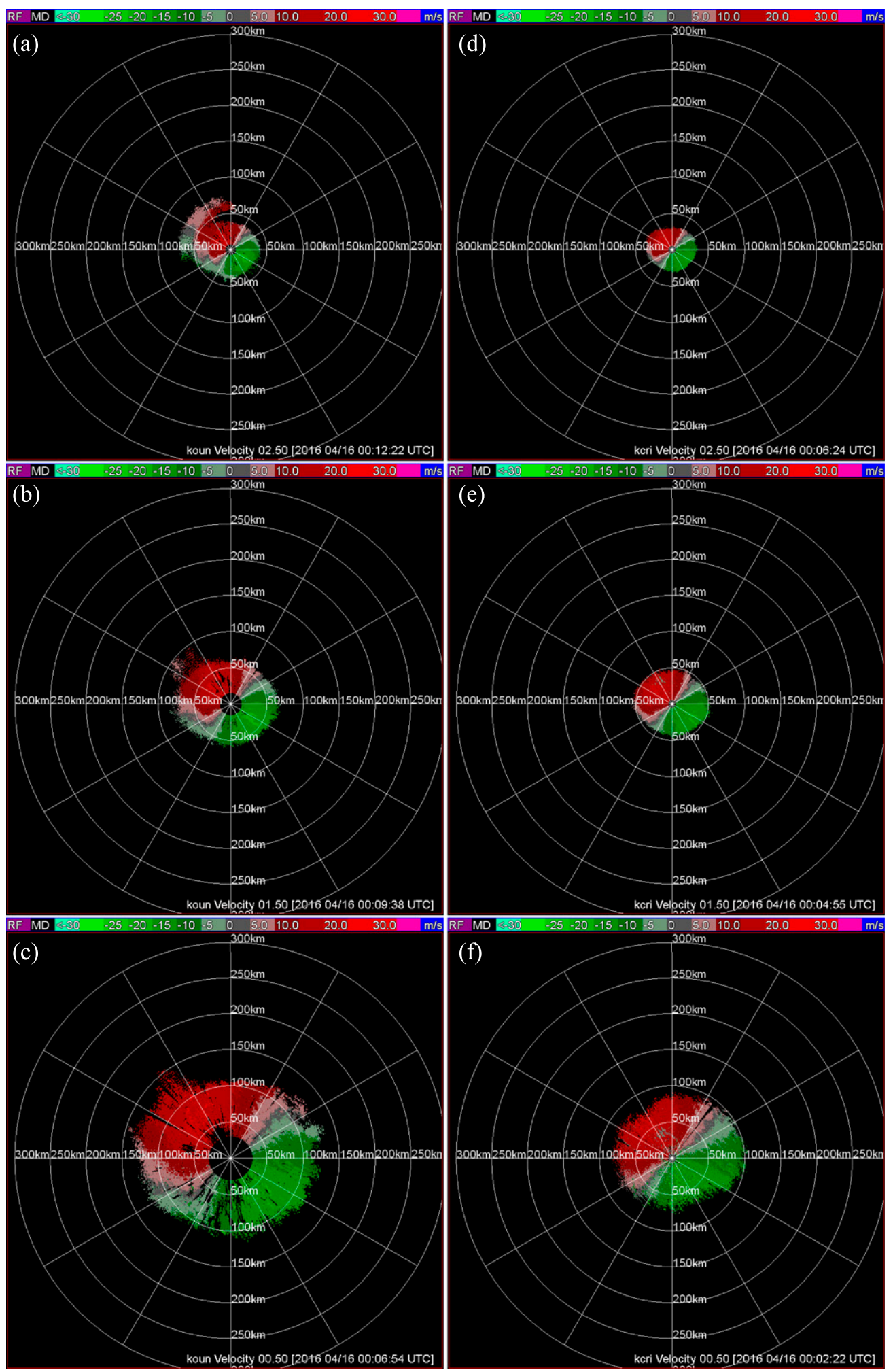

FIG. 2. (a)-(c) Images of dealiased velocities produced by the SA-VDA (described in section 2) from the KOUN scanned raw velocities in Figs. 1a-c. (d)-(f) As in (a)-(c), but produced from the KCRI scanned raw velocities in Figs. 1d-f. The black hole around the radar site in each panel shows the radial range within which the raw velocities are removed before dealiasing (for the reason explained in section $3 a$ ). 

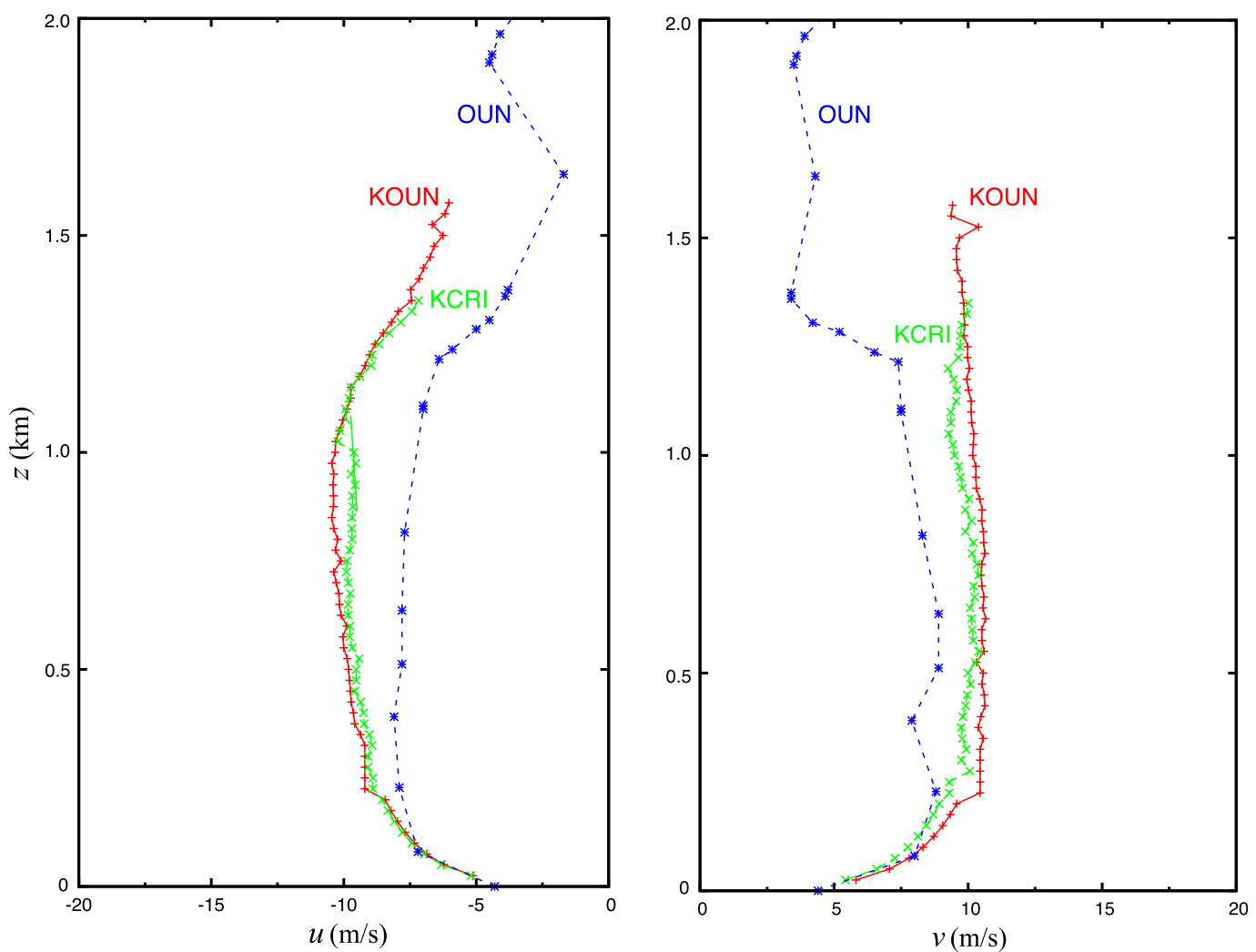

FIG. 3. Vertical profiles of VAD wind components (left) $u$ and (right) $v$ computed, as by-products of dealiasing, by using the dealiased velocities within $80-\mathrm{km}$ radial range on the three sweeps in Figs. 2a-c from KOUN (in red) and on the three sweeps in Figs. 2d-f from KCRI (in green) against the vertical profiles of rawinsonde winds (in blue) observed at 0000 UTC 16 Apr 2016 from the operational rawinsonde site (named OUN, about $7 \mathrm{~km}$ from KOUN radar site) in Norman, OK.

became very noisy near the radar, especially inside of $30 \mathrm{~km}$ on $0.5^{\circ}, 15 \mathrm{~km}$ on $1.5^{\circ}$, and $5 \mathrm{~km}$ on $2.5^{\circ}$. These data are removed prior dealiasing, and shown by the black holes on the three sweeps around the radar site in Figs. 2a-c. On the other hand, the raw level-II velocity data from KCRI were not subject to this problem, so only data inside of $5 \mathrm{~km}$ are blocked and thus lost as shown by the small black holes around the radar site in Figs. 2d-f.

Using the velocity-azimuth display (VAD) technique (Lhermitte and Atlas 1961; Browning and Wexler 1968), vertical profiles of horizontal wind $(u, v)$ are computed (as by-products of dealiasing) by using the dealiased velocities within $80-\mathrm{km}$ radial range on the three sweeps from KOUN (Figs. 2a-c) versus those from KCRI (Figs. 2d-f). The computed VAD wind profiles are plotted in Fig. 3 and compared to rawinsonde winds observed at 0000 UTC 16 April 2016 from the operational rawinsonde site (WMO Station Identifier number 72357 , about $7 \mathrm{~km}$ from KOUN radar site and named OUN in this paper) in Norman, Oklahoma. The vertical profiles of VAD wind component $u$ (or $v$ ) from the two radars are very similar (Fig. 3), and both reasonably close to the vertical profile of rawinsonde wind component $u$ (or $v$ ), but the VAD winds from KOUN go up to $1.6 \mathrm{~km}$, those from KCRI go up to only1.3 km.

\section{b. Results obtained under rainy weather condition}

Raw velocities scanned from KOUN using the new scan mode under a rainy weather condition around 0000 UTC 18 April 2016 are shown in Figs. 4a-c (at 2.5 $1.5^{\circ}$, and $0.5^{\circ}$ ) in comparison with those in Figs. $4 \mathrm{~d}-\mathrm{f}$ scanned from KCRI (at $2.5^{\circ}, 1.3^{\circ}$, and $0.5^{\circ}$ ) around the same time. Using the new scan mode, the maximum range increases about $50 \%$. Velocity aliasing becomes a problem near each white " $A$ " that marks an aliasedvelocity area in Figs. 4a-c, where aliasing appears in patches due to presumed turbulent winds. Velocities from KCRI are also aliased for the same reasons in a small area also marked by a white "A" on each sweep (Figs. 4d-f).

Using the SA-VDA, the aliased velocities from KOUN are mostly corrected in contiguous areas around the radar (Figs. 5a-c). Velocities in isolated 

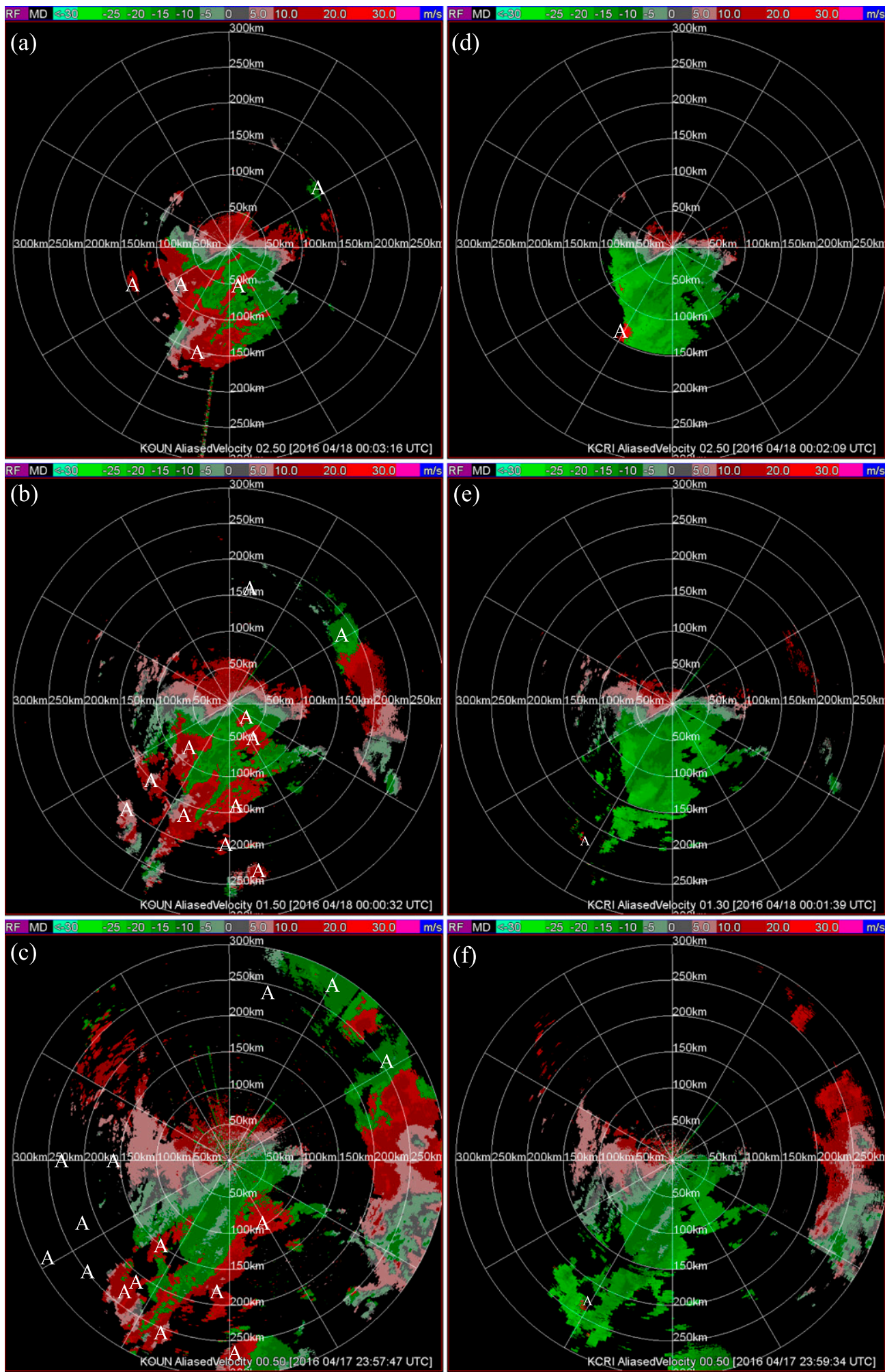

FIG. 4. (a)-(c) As in Figs. 1a-c, but scanned under a rainy weather condition around 0000 UTC 18 Apr 2016. (d)-(f) As in Figs. $1 \mathrm{~d}-\mathrm{f}$, but scanned at $2.5^{\circ}, 1.3^{\circ}$, and $0.5^{\circ}$ under the same rainy weather condition around the same time as in (a)-(c). 

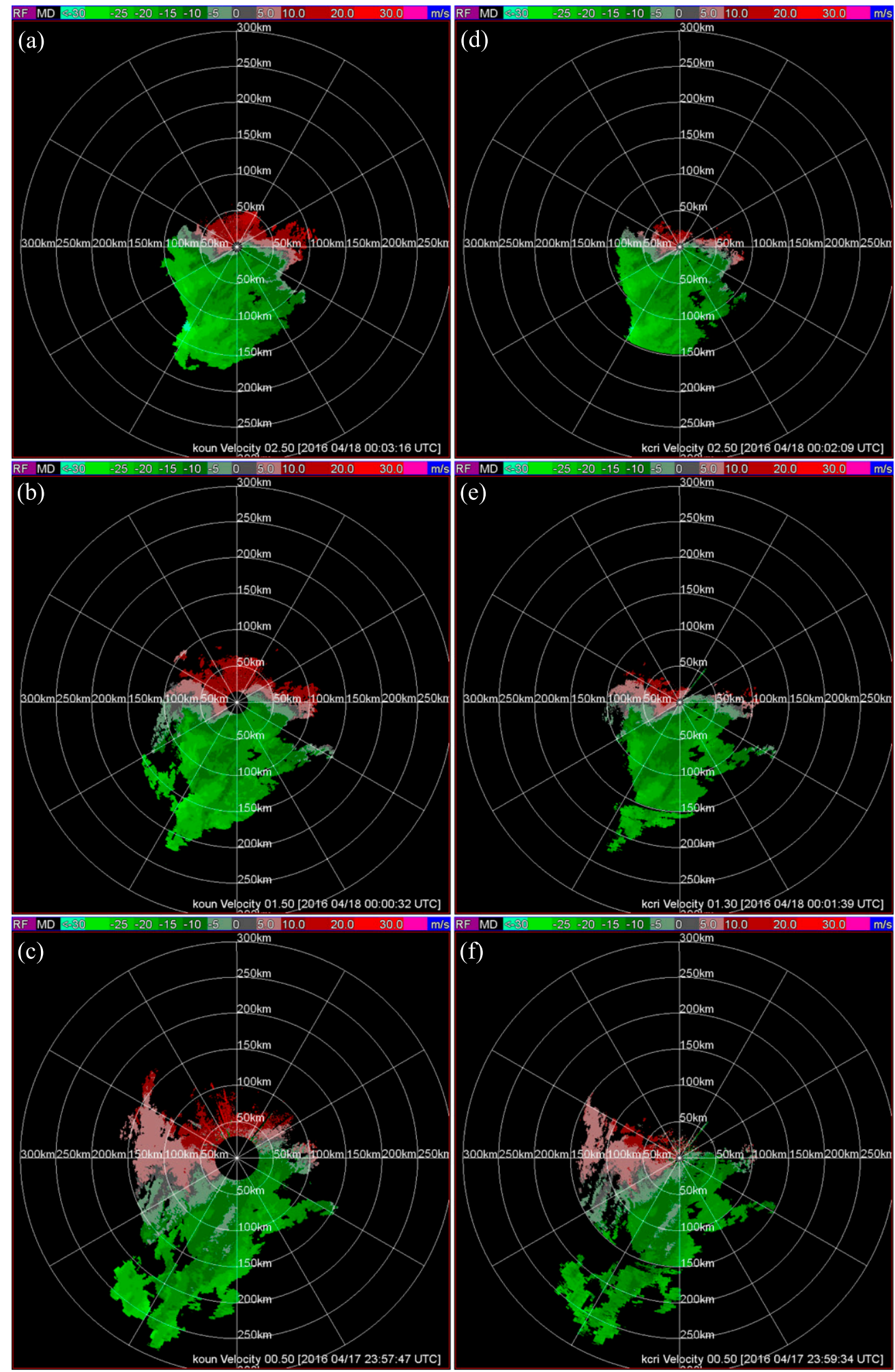

FIG. 5. As in Fig. 2, but produced from the raw velocities in Fig. 4. 

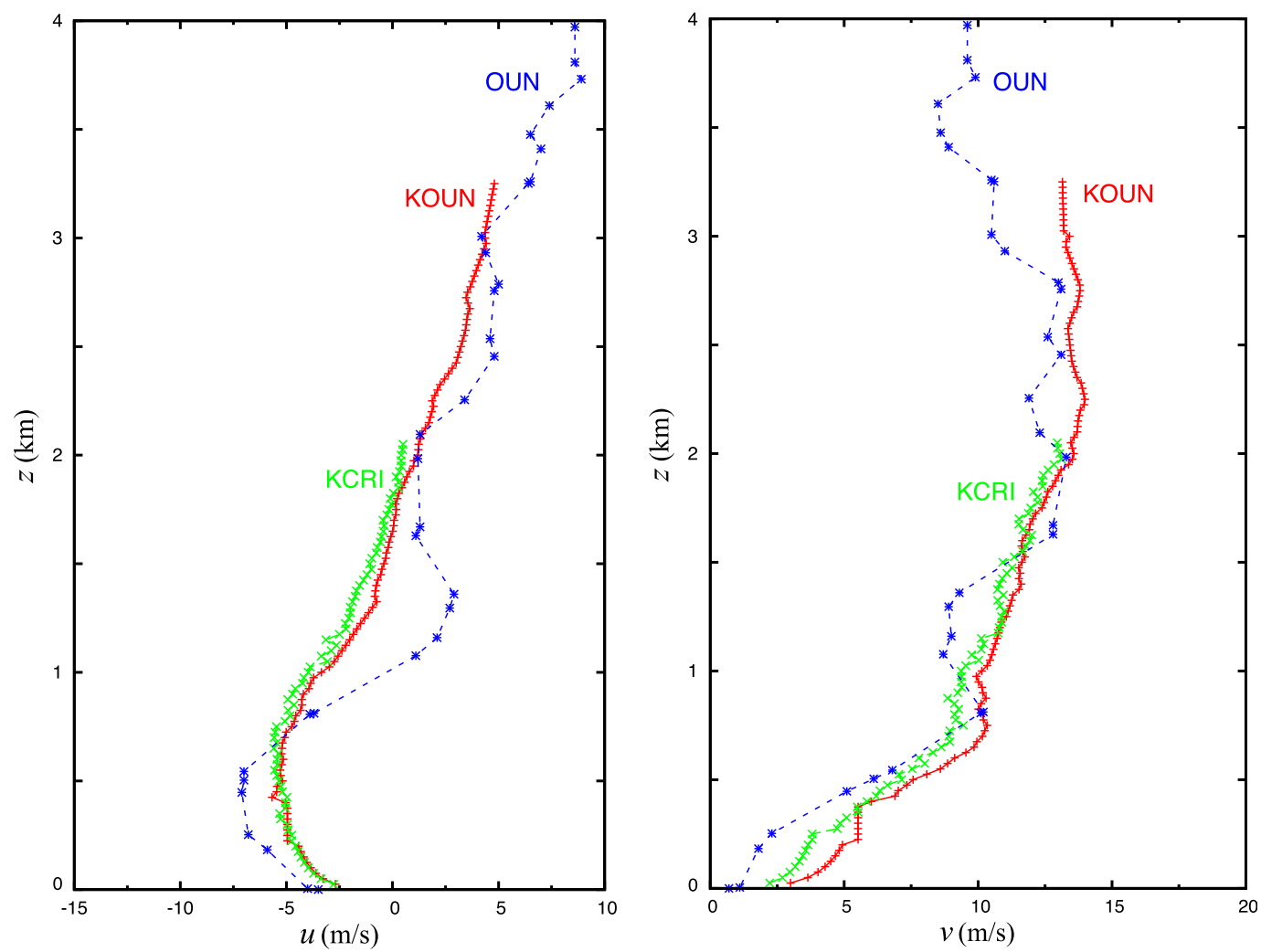

FIG. 6. As in Fig. 3, but computed by using the dealiased velocities in Fig. 5 against the vertical profiles of rawinsonde winds observed at 0000 UTC 18 Apr 2016 from OUN.

areas (associated with precipitation) east of the radar are all discarded by the SA-VDA, because the continuity check (in the second step of dealiasing) cannot pass the large gaps [by 40 gates and 5 beams or more, as stated in the last paragraph of section 2 of Xu et al. (2013)] between the main and isolated data areas. This is a limitation of the SA-VDA, and is also seen in Figs. 5d-f for the velocities from KCRI.

Figure 6 shows the VAD winds computed with dealiased velocities within $80 \mathrm{~km}$ from KOUN (Figs. 5a-c) versus those from KCRI (Figs. 5d-f). Rawinsonde winds observed at 0000 UTC 18 April 2016 from OUN are also plotted in Fig. 6. The VAD winds from the two radars are very close to each other and both close to the rawinsonde winds, but KOUN winds are retrieved through a greater depth $(3.3 \mathrm{~km})$ than winds from KCRI $(2.1 \mathrm{~km})$.

\section{c. Results obtained under conditions of clear-air and scattered precipitation}

Raw velocities from KOUN using the new scan mode under conditions of clear-air and scattered precipitation around 0000 UTC 20 April 2016 are shown in Figs. $7 \mathrm{a}-\mathrm{c}$ (at $2.5^{\circ}, 1.5^{\circ}$, and $0.5^{\circ}$ ) versus those from KCRI (Figs. 7d-f). The new scan mode nearly doubled the range of clear-air data coverage around the radar. As before, aliasing around KOUN is marked by the white "A"s. Beyond $50 \mathrm{~km}$ west and southwest of the radar, aliasing is seen in many irregular patches in areas of scattered precipitation (Figs. 7a-c).

Using the SA-VDA, aliasing appearing in KOUN are corrected not only in the clear-air area near and around the radar but also in areas of precipitation that are contiguous with the clear-air area (Figs. 8a-c). As before, velocities in isolated data "islands" are all discarded (Fig. 8). Note that in the KCRI data at $1.3^{\circ}$ and $2.5^{\circ}$, the scattered, noncontiguous areas are largely discarded (Figs. 8e,f compared with Figs. 7e,f).

Figure 9 shows VAD winds for KOUN (Figs. 8a-c) versus KCRI (Figs. 8d-f) all compared to the nearby, contemporaneous rawinsonde winds. As before, both VAD profiles are very similar, but KOUN is twice as deep as KCRI $(1.6 \mathrm{~km}$ vs $0.8 \mathrm{~km})$. Similar results and comparisons are obtained (not shown) for velocities from the two radars under similar conditions on 17 April 2016. 

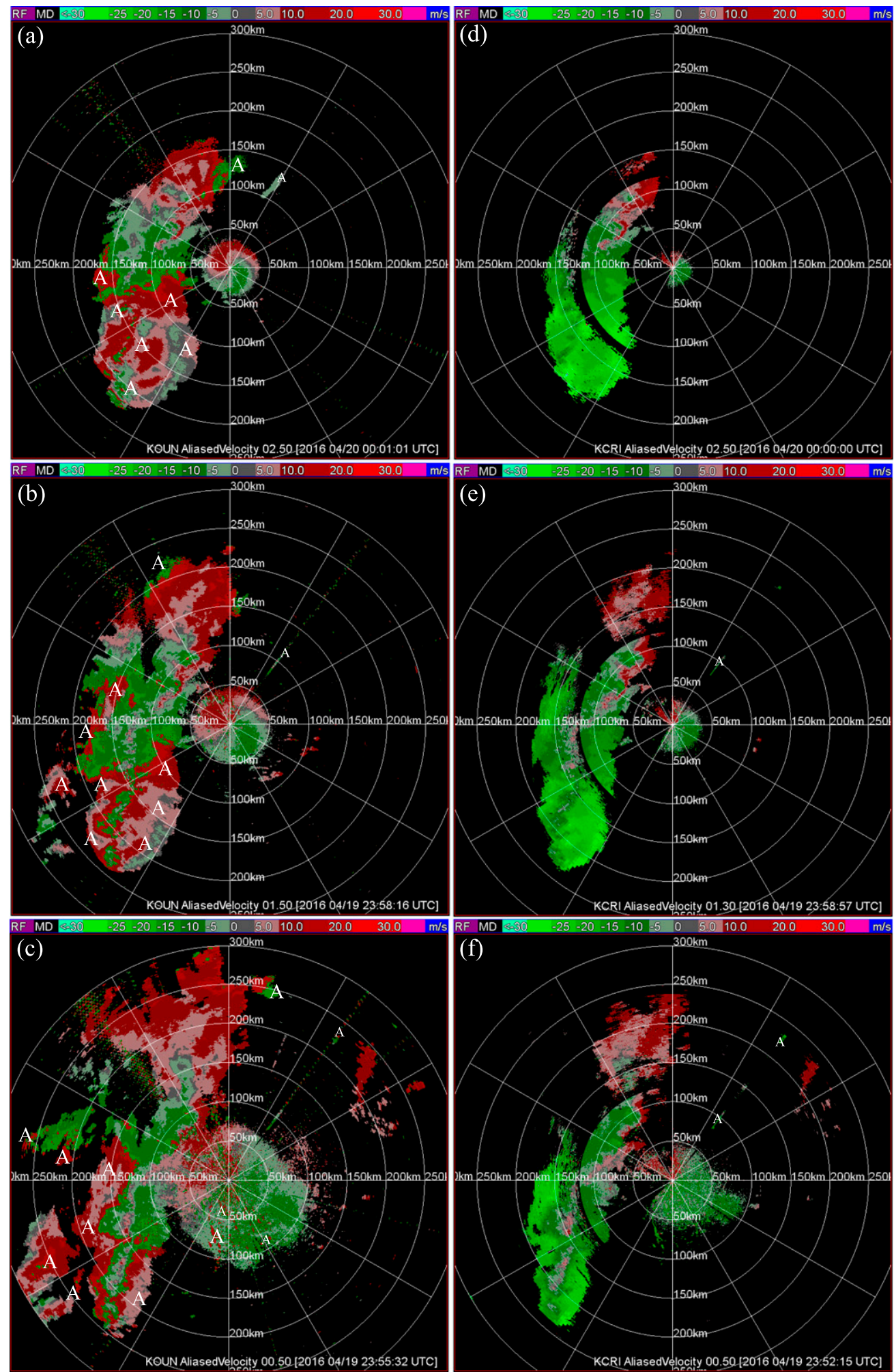

FIG. 7. As in Fig. 4, but scanned under conditions of clear-air and scattered precipitation around 0000 UTC 20 Apr 2016. 

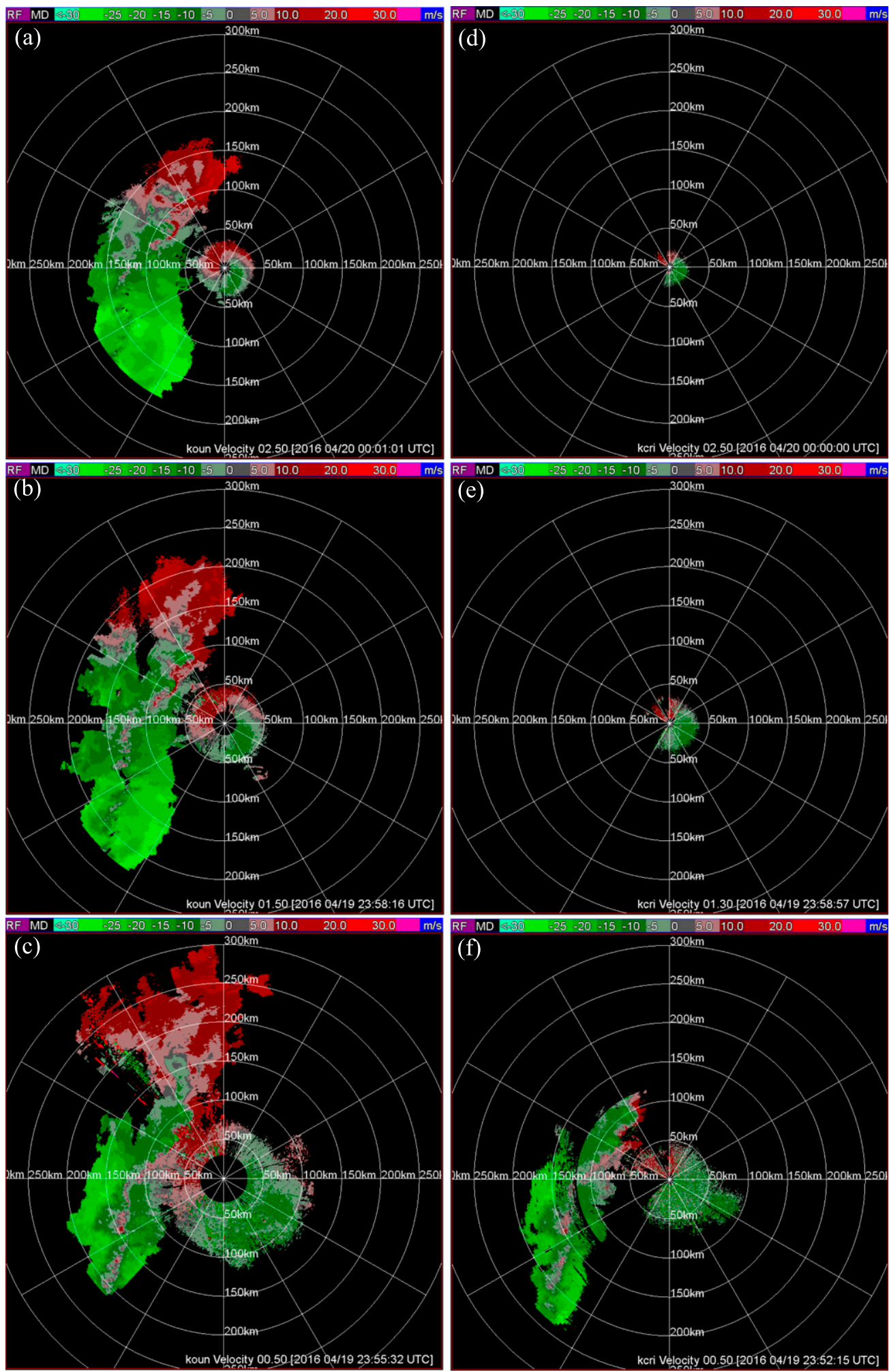

FIG. 8. As in Fig. 2, but produced from the raw velocities in Fig. 7 

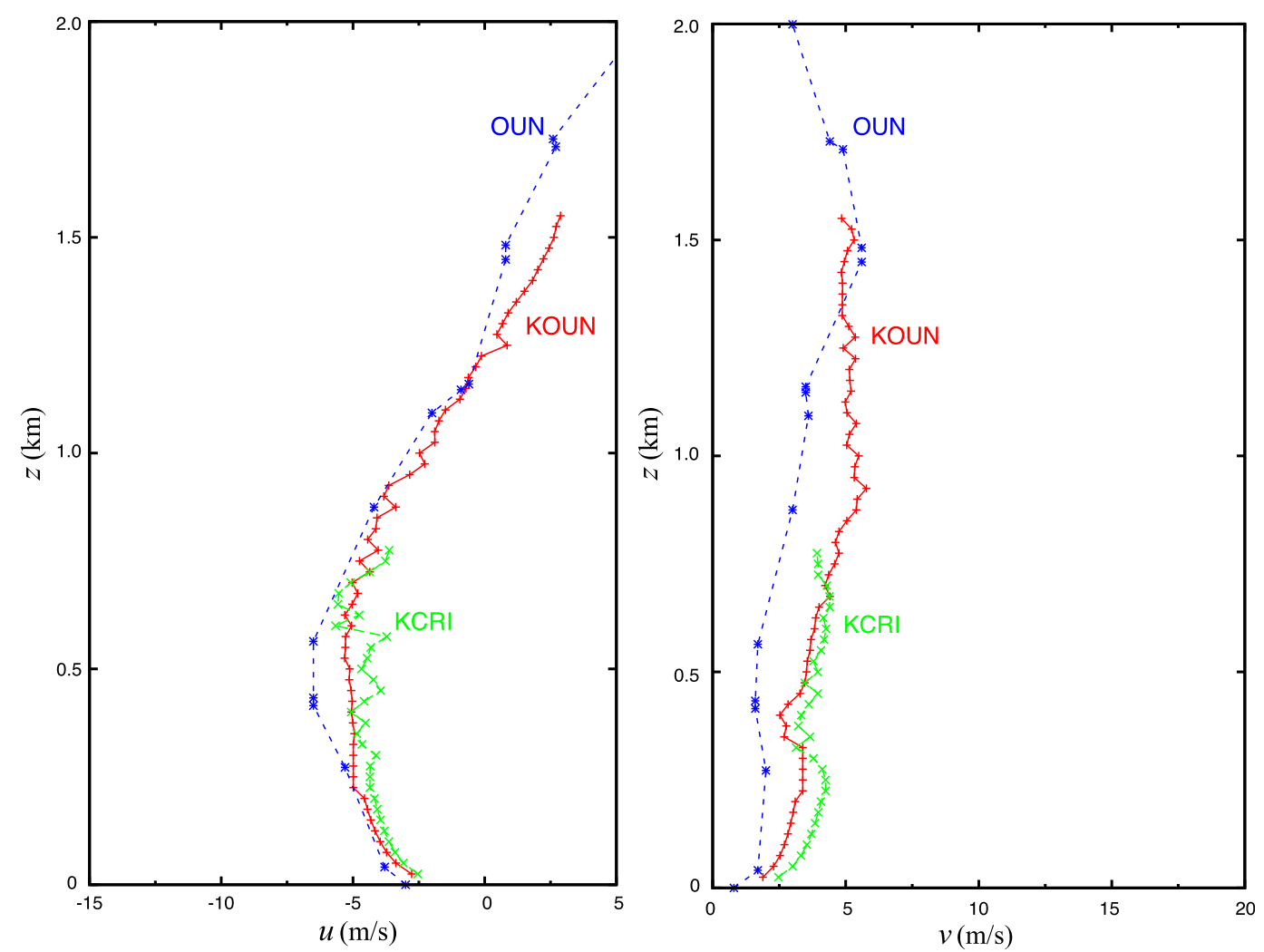

FIG. 9. As in Fig. 6, but computed by using the dealiased velocities in Fig. 8 against the vertical profiles of rawinsonde winds observed at 0000 UTC 20 Apr 2016.

\section{Results obtained with supplemental high-PRF velocity data}

a. Results obtained with supplemental dealiased high-PRF velocity data

In the previous section, the SA-VDA is applied successfully to KOUN using the new scan mode for similar cases under different weather conditions. However, in many cases KOUN velocities are too noisy near the radar for the AR-Var to produce reference velocities for the reference check in the first step of dealiasing [see section 3 of $\mathrm{Xu}$ et al. (2013)]. Thus, the raw velocities are all discarded by the SA-VDA. The KCRI velocities are noisy but not as much as KOUN, so the SA-VDA can still produce good reference velocities and successfully perform dealiasing. For these cases, a modification is made to the SA-VDA that uses dealiased velocities from KCRI as proxy references for the reference check applied to the raw velocities from KOUN. This is an effective strategy, although the proxy reference velocity data contain data-void holes (as shown in Figs. 11d-f) and thus have different spatial characteristics than the continuous reference velocity fields generated from the original-VCP31 long-pulse raw velocity observations [see Fig. $2 \mathrm{~b}$ of $\mathrm{Xu}$ and Nai (2012)].

Figures $10 \mathrm{a}-\mathrm{c}$ show the raw velocities scanned from KOUN (at $2.5^{\circ}, 1.5^{\circ}$, and $0.5^{\circ}$ ) using the new scan mode in clear-air conditions around 12 UTC 16 April 2016 compared to KCRI (Figs. 10d-f). The new scan increases the range of data coverage more than $200 \%$, but KOUN velocity data become very noisy, much worse than those from KCRI. In particular, as shown in Fig. 10c, the raw velocities from KOUN are not only very noisy but also aliased twice around $100 \mathrm{~km}$ south of the radar, where the raw velocities from KCRI display only scattered aliasing (Fig. 10f). In this case, the SA-VDA can produce reasonable results from KCRI (Figs. 10d-f) but not from KOUN (Figs. 10a-c). However, when using the dealiased velocities from KCRI (shown in Figs. 11d-f) as proxy references, the KOUN velocities are properly dealiased. Figures 11a-f illustrate the improvements obtained on data from KOUN versus similar data from KCRI.

Figure 12 shows VAD profiles from both KOUN and KCRI compared to rawinsonde winds for 0000 UTC 

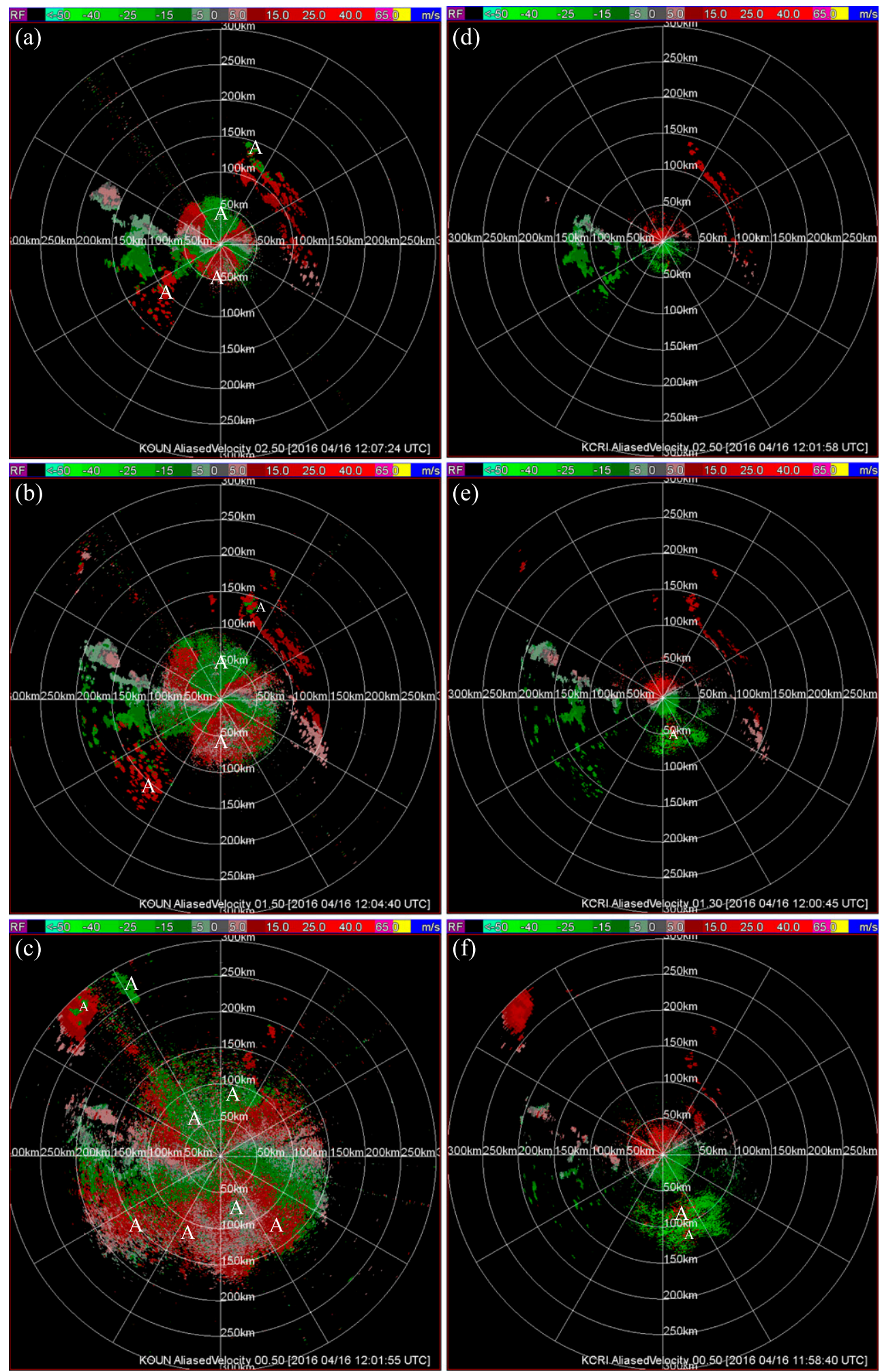

FIG. 10. As in Fig. 7, but scanned under a clear-air weather condition around 1200 UTC 16 Apr 2016. 

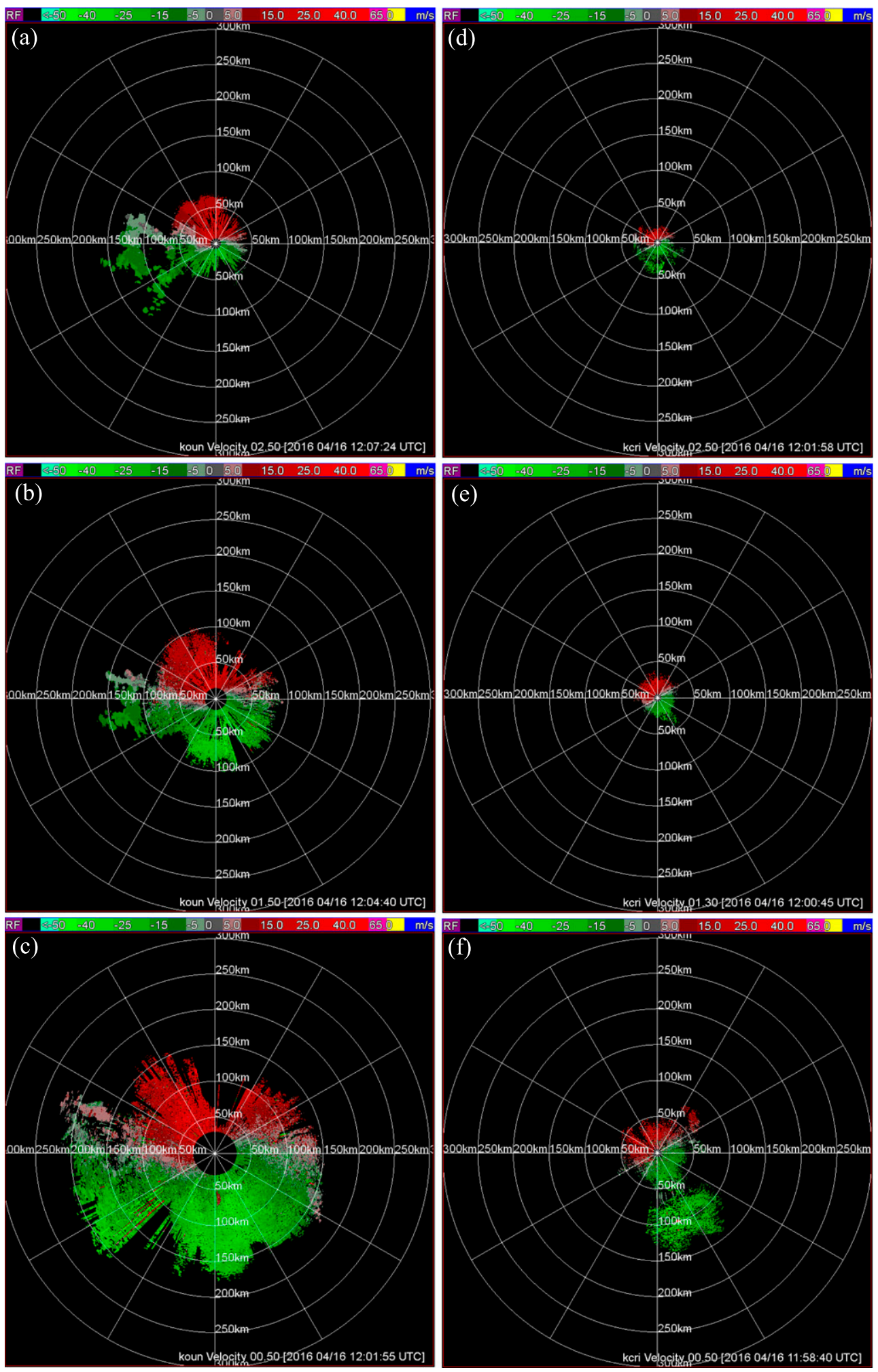

FIG. 11. As in Fig. 8, but produced by the first-type modified dealiasing (described in section 2) from the raw velocities in Fig. 10. 

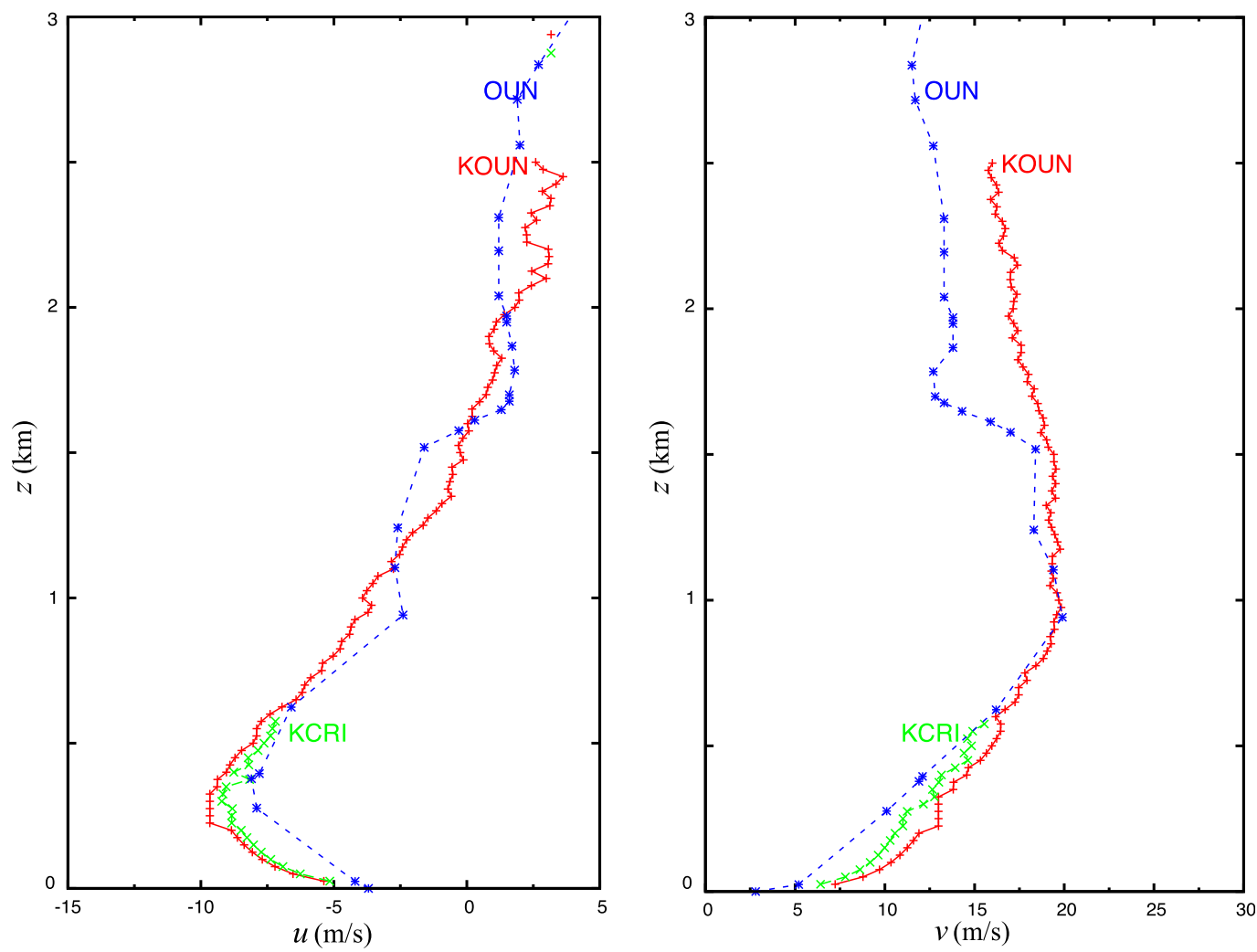

FIG. 12. As in Fig. 9, but computed by using the dealiased velocities in Fig. 11 against the vertical profiles of rawinsonde winds observed at 1200 UTC 16 Apr 2016.

20 April 2016. As before, KOUN data extend up further than KCRI data $(2.5 \mathrm{~km}$ vs $0.6 \mathrm{~km})$.

\section{b. Results obtained with supplemental undealiased raw high-PRF velocity data}

Occasionally, velocities from KCRI become too noisy near the radar and/or too sparse to be processed by the SA-VDA. Yet, these raw velocities were essentially free of aliasing and so may be used directly (except for noisy data near the radar) as proxy references for the reference check applied to the raw velocities from KOUN.

Figures $13 \mathrm{a}-\mathrm{c}$ show the raw velocities scanned from KOUN (at $2.5^{\circ}, 1.5^{\circ}$, and $0.5^{\circ}$ ) using the new scan mode under conditions of clear-air and scattered precipitation around 1200 UTC 18 April 2016. Figures 13d-f show the raw velocities from KCRI (at $2.5^{\circ}, 1.3^{\circ}$, and $0.5^{\circ}$ ) around the same time. The new scan increases the data coverage significantly in such conditions. Figures $13 d-f$ show that aliasing was not a problem for KCRI. Note that the precipitation VCP12 was used here by KCRI, but any precipitation VCPs with sufficiently high PRFs could also be used in this case. Thus, KCRI data can be used directly as proxy references for the reference check applied to KOUN raw velocities via the second-type modification. Note that the level-II raw velocity data from KOUN became very noisy near the radar and these near-radar data are removed before dealiasing (for the reason explained in section 3a), so the near-radar noisy raw velocity data from KCRI are not used and thus also removed (as shown by the enlarged black holes around the KCRI radar site in Figs. 14e and 14f vs those in Figs. $13 \mathrm{e}$ and $13 \mathrm{f}$ ). Figures $14 \mathrm{a}-\mathrm{c}$ show that velocity data from KOUN are well dealiased in both clear air and areas of precipitation using the second-type modified dealiasing. Results are particularly clearly shown in Fig. $14 \mathrm{a}$, where KOUN velocities at $2.5^{\circ}$ elevation are successfully dealiased when using data from KCRI as proxy references.

Similar to prior figures, Fig. 15 shows VAD profiles from both radars compared with contemporaneous, nearby rawinsonde data. For 1200 UTC 18 April 2016 as before, the VAD winds from KOUN extend over a deeper layer (up to $0.7 \mathrm{~km}$ ) than those from KCRI (up to $0.35 \mathrm{~km}$ ).

Similar results and comparisons are obtained (not shown) for velocities from both radars under similar conditions around 0000 UTC 19 April 2016. The VAD profiles are deeper for KOUN than for KCRI, but compare well 

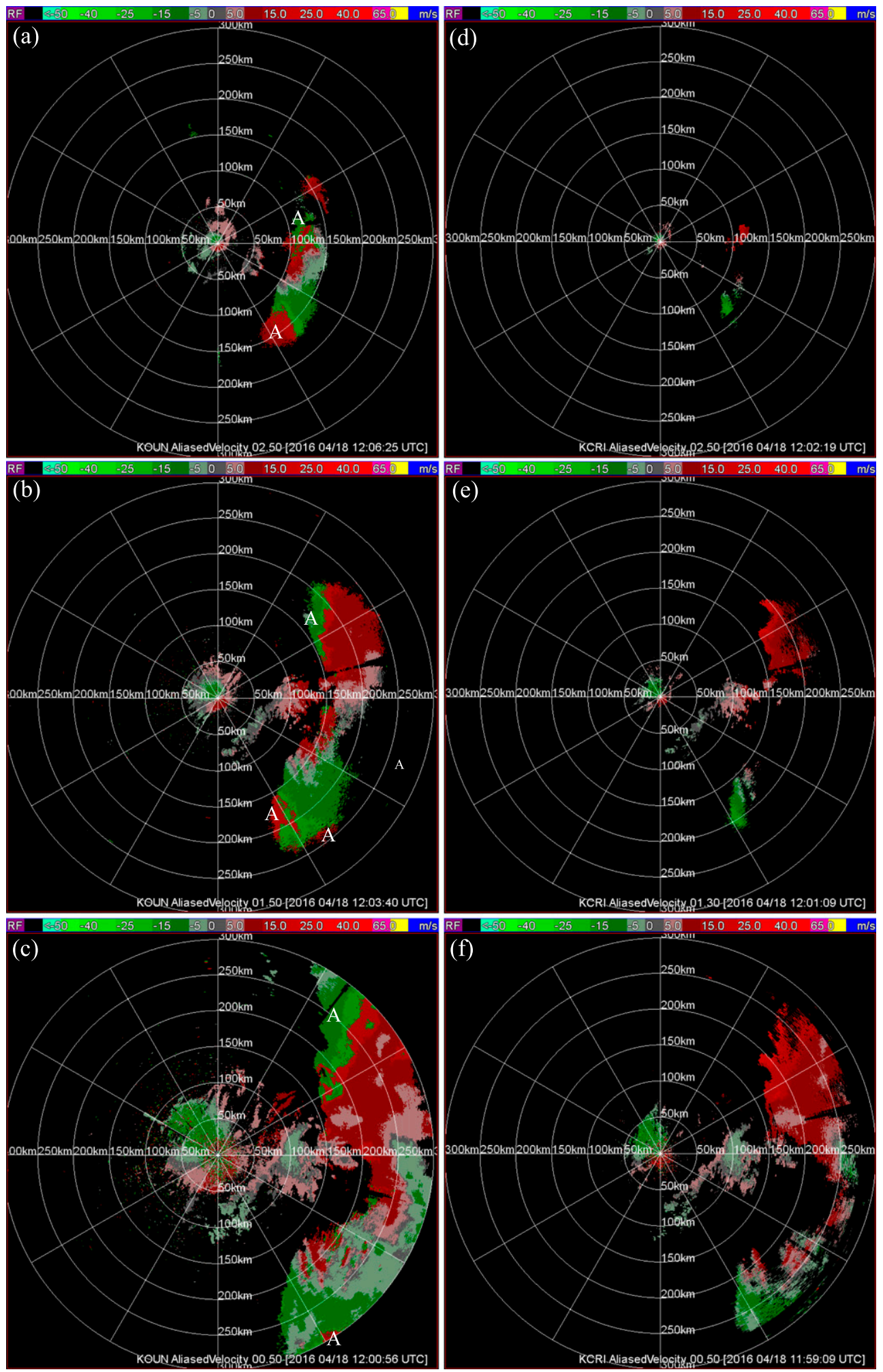

FIG. 13. As in Fig. 10, but scanned under conditions of clear-air and scattered precipitation around 1200 UTC 18 Apr 2016. 

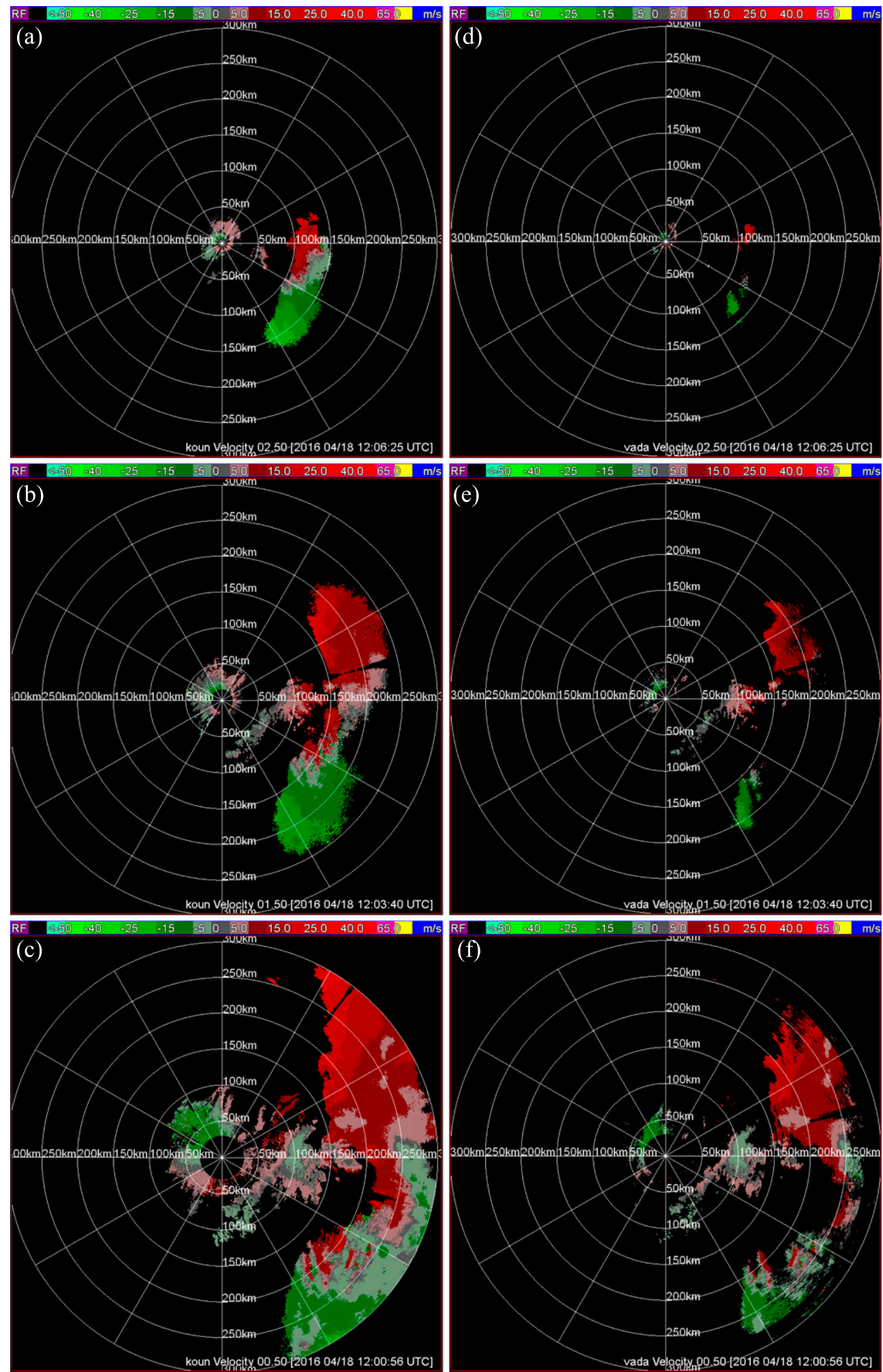

FIG. 14. (a)-(c) As in Figs. 11a-c, but produced by the second-type modified dealiasing (described in section 2) from the raw velocities in Figs. 13a-c. (d)-(f) As in Figs. 13d-f, but used as references with the raw velocities removed within the same radial ranges as those used to remove KOUN raw velocities before dealiasing (as shown by the enlarged black holes around the KCRI radar site in comparison with those in Figs. 13e-f). 

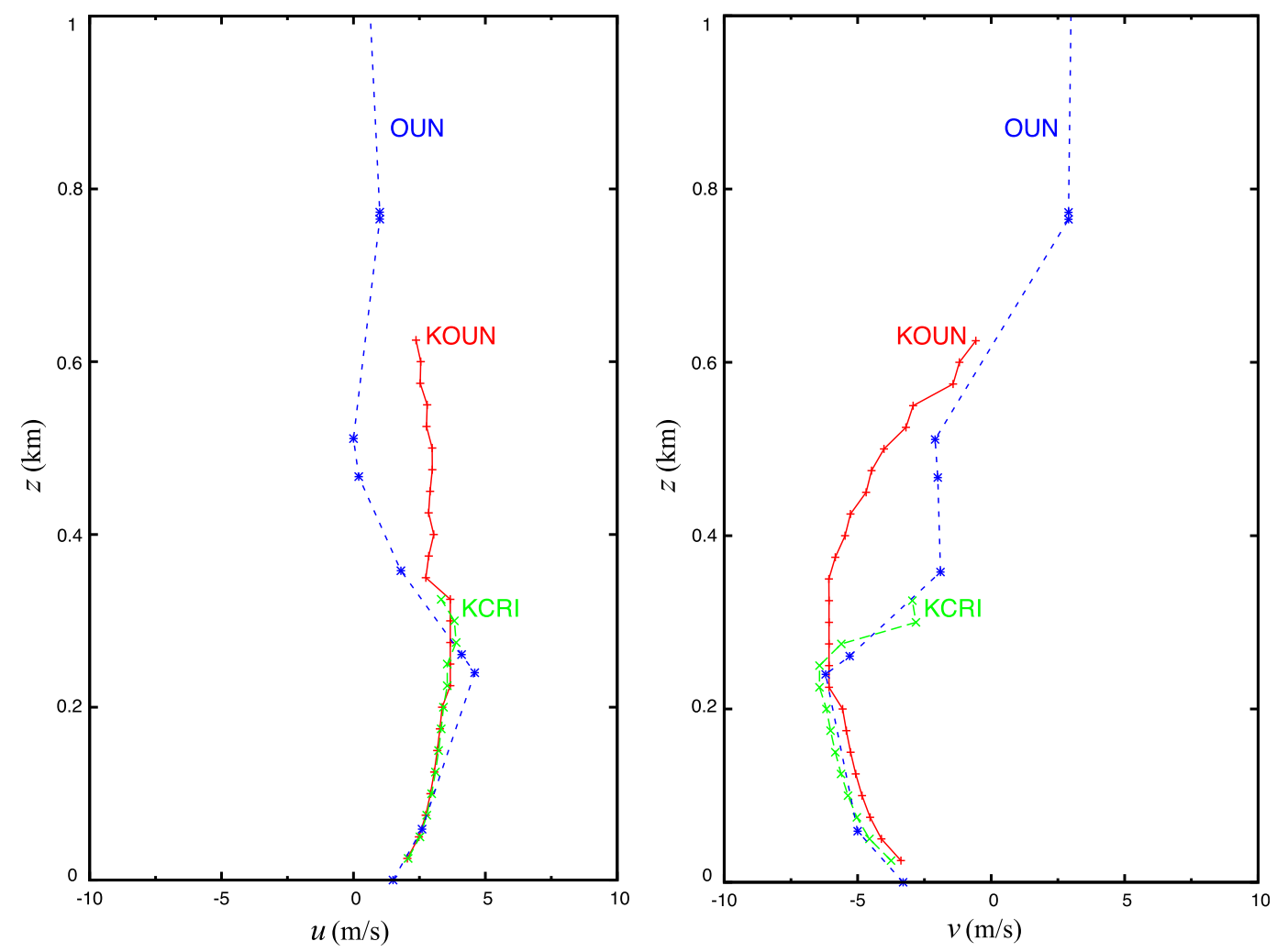

FIG. 15. As in Fig. 12, but computed by using the dealiased velocities in Fig. 14 against the vertical profiles of rawinsonde winds observed at 1200 UTC 18 Apr 2016.

where available from both radars, and the KOUN VAD profile compared well with the rawinsonde wind profile.

\section{Conclusions}

The VCP31 (or VCP32) scan mode currently used for clear-air by the KOUN WSR-88D test bed radar at NSSL is modified by slowing the antenna rotation rate to increase the dwell time (or by using long pulses and slowing the antenna rotation rate), which in turn increases radar sensitivity. This enhanced sensitivity increases spatial coverage of velocity. With adequate data quality control, these data are useful in data assimilation that should improve numerical weather predictions.

Using the newly modified scan mode, Doppler velocities from KOUN over a limited period (16-20 April 2016) are shown to have significantly enhanced data coverage compared to the standard VCP32 from KCRI (the operational test bed radar located only $315 \mathrm{~m}$ from KOUN). However, since the Nyquist velocity is reduced to $12.53 \mathrm{~m} \mathrm{~s}^{-1}$ due to the low PRF used in the new scan, raw velocities scanned from KOUN are seriously aliased and cause enormous difficulties in dealiasing.

To mitigate Doppler velocity aliasing problems caused by the reduced Nyquist velocity, the recently developed dealiasing technique (Xu et al. 2013) for operational WSR-88Ds is modified back into a stand-alone version, called SA-VDA, in which the reference velocities are produced by applying the alias-robust variational analysis (AR-Var; Xu and Nai 2013) to KOUN raw velocities (see section 2). This SA-VDA is applied successfully to raw velocities collected from KOUN for various time periods under different weather conditions (see section 3) but not for all conditions over the entire test period (from 16 to 20 April).

While effective, the SA-VDA fails when raw velocities from KOUN become very noisy. In these conditions, raw velocities from KCRI can still be used to produce dealiased velocities. These dealiased velocities are then used as proxy references for the reference check applied to the raw velocities from KOUN. This modification is effective as long as the SA-VDA can dealias raw velocities from KCRI (see section 4a). This modification mimics a dual-PRF approach similar to the multi-PRF approach developed by Conway et al. (1997) and Zittel et al. (2008) but with two important differences. First, it uses two (instead of three) different PRFs. In particular, it uses a high PRF (with short pulses) first to scan the entire sweep at a fixed elevation angle and then uses a low PRF (with long pulses which reduce the 


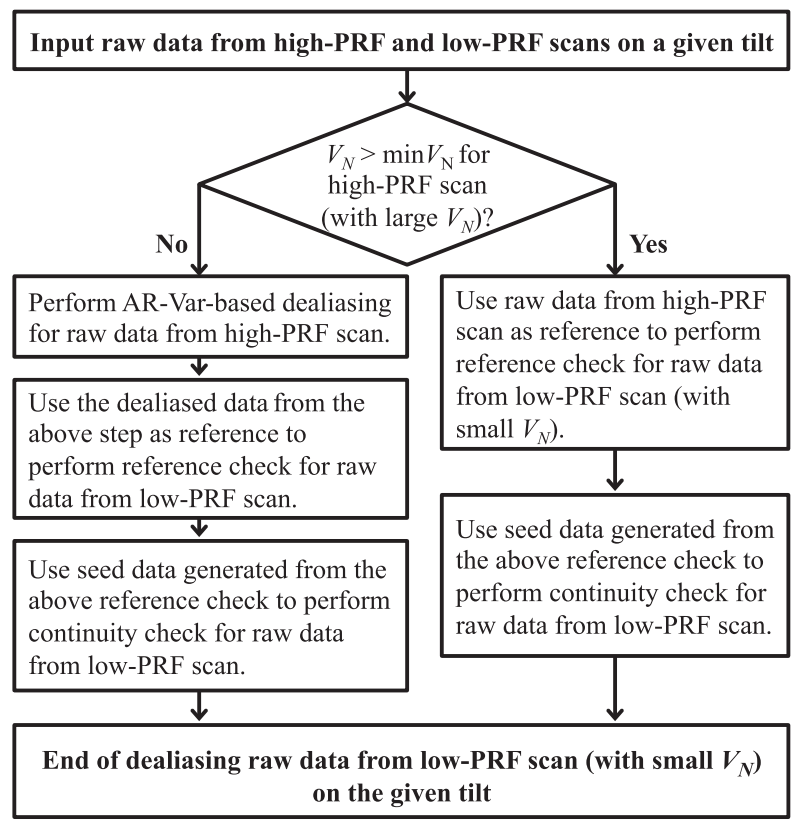

FIG. 16. Flowchart of algorithm procedures for dealiasing raw velocities from proposed dual-PRF scans. Here, $\min V_{N}$ is the minimally required Nyquist velocity to ensure the raw velocities from the high-PRF scan to be free of alias or almost so. The value of $\min V_{N}$ can and will be determined empirically for each given type of weather condition. The algorithm procedure for the case of $V_{N}$ not larger (or larger) than $\min V_{N}$ along the left (or right) column corresponds to the first (or second) type of modification of the SA-VDA as described in section 2.

Nyquist interval) to scan the entire sweep again at the same fixed elevation angle. Second, the two scans are not dealiased simultaneously. Instead, the first scan is dealiased first by using the SA-VDA, then using the dealiased velocities from the first scan as references for the second scan. In this case, the algorithm becomes stand-alone again because reference velocities are obtained internally from KOUN (rather than externally from KCRI).

Doppler velocities from KCRI are occasionally too noisy and/or too sparse for the SA-VDA to be successful. Yet, these Doppler velocities were generally free of aliasing problems and thus can be used directly as proxy references for the reference check applied to KOUN raw velocities. This modification is very effective in producing dealiased velocities (see section $4 b$ ). This second type of modification mimics another type of dual-PRF dealiasing that is similar to the first type described above but requires a large enough Nyquist interval to alleviate most aliasing issues in the first scan.

To test and implement the above two types of dualPRF dealiasing (mimicked by the two-types of modifications experimented in this paper), new dual-PRF scans are being designed to collect clear-air Doppler velocity data from KOUN. The algorithm procedures for dealiasing the collected raw velocity data on each tilt are sketched by the flowchart in Fig. 16. The dealiased data will be used to explore the full potential of the new scan mode (designed in this paper and further incorporated into dual-PRF scans).

Acknowledgments. We are thankful to David Zittel at the NWS Radar Operations Center and two anonymous reviewers for their constructive comments and suggestions, and to Dr. Kim Elmore at NSSL for reviewing the original manuscript internally. The research work was supported by the Warn-on-Forecast project at NSSL and the ONR Grant N000141712375 to the University of Oklahoma (OU). Funding was also provided to CIMMS by NOAA/Office of Oceanic and Atmospheric Research under NOAA-OU Cooperative Agreement NA11OAR4320072, U.S. Department of Commerce.

\section{REFERENCES}

Brotzge, J., and W. Donner, 2013: The tornado warning process: A review of current research, challenges, and opportunities. Bull. Amer. Meteor. Soc., 94, 1715-1733, https://doi.org/ 10.1175/BAMS-D-12-00147.1.

Browning, K. A., and R. Wexler, 1968: The determination of kinematic properties of a wind field using Doppler radar. J. Appl. Meteor., 7, 105-113, https://doi.org/10.1175/15200450(1968)007<0105:TDOKPO > 2.0.CO;2.

Conway, J. W., K. D. Hondl, and M. D. Eilts, 1997: Minimizing the Doppler dilemma using a unique redundant scanning strategy and multiple pulse repetition frequency dealiasing algorithm. Preprints, 28th Conf. on Radar Meteor, Austin, TX, Amer. Meteor. Soc., 315-316.

Crum, T. D., and R. L. Alberty, 1993: The WSR-88D and the WSR88D operational support facility. Bull. Amer. Meteor. Soc., 74 1669-1687, https://doi.org/10.1175/1520-0477(1993)074<1669: TWATWO $>2.0 . \mathrm{CO} ; 2$.

—, R. E. Saffle, and J. W. Wilson, 1998: An update on the NEXRAD program and future WSR-88D support to operations. Wea. Forecasting, 13, 253-262, https://doi.org/10.1175/ 1520-0434(1998)013<0253:AUOTNP > 2.0.CO;2.

Elmore, K. L., P. L. Heinselman, and D. J. Stensrud, 2012: Using WSR-88D data and insolation estimates to determine convective boundary layer depth. J. Atmos. Oceanic Technol., 29, 581-588, https://doi.org/10.1175/JTECH-D-11-00043.1.

Gao, J., K. K. Droegemeier, J. Gong, and Q. Xu, 2004: A method for retrieving mean horizontal wind profiles from singleDoppler radar observations contaminated by aliasing. Mon. Wea. Rev., 132, 1399-1409, https://doi.org/10.1175/1520-0493132.1.1399.

Gong, J., L. Wang, and Q. Xu, 2003: A three-step dealiasing method for Doppler velocity data quality control. J. Atmos. Oceanic Technol., 20, 1738-1748, https://doi.org/10.1175/15200426(2003)020<1738:ATDMFD>2.0.CO;2.

Hardegree, S. P., S. S. Van Vactor, D. H. Levinson, and A. H. Winstral, 2008: Evaluation of NEXRAD radar precipitation products for natural resource applications. Rangeland Ecol. Manage., 61, 346-353, https://doi.org/10.2111/07-036.1. 
Heinselman, P. L., D. J. Stensrud, R. M. Hluchan, P. L. Spencer, P. C. Burke, and K. L. Elmore, 2009: Radar reflectivity-based estimates of mixed layer depth. J. Atmos. Oceanic Technol., 26, 229-239, https://doi.org/10.1175/2008JTECHA1091.1.

Jiang, Y., Q. Xu, P. Zhang, K. Nai, and L. Liu, 2013: Using WSR88D polarimetric data to identify bird-contaminated Doppler velocities. Adv. Meteor., 2013, 769275, https://doi.org/10.1155/ 2013/769275.

Jing, Z., and G. Wiener, 1993: Two-dimensional dealiasing of Doppler velocities. J. Atmos. Oceanic Technol., 10, 798-808, https://doi.org/10.1175/1520-0426(1993)010<0798:TDDODV $>$ 2.0.CO;2.

Lakshmanan, V., T. Smith, G. Stumpf, and K. Hondl, 2007: The warning decision support system-integrated information. Wea. Forecasting, 22, 596-612, https://doi.org/ 10.1175/WAF1009.1.

Lhermitte, R. M., and D. Atlas, 1961: Precipitation motion by pulse Doppler. Preprints, Ninth Weather Radar Conf., Kansas City, MO, Amer. Meteor. Soc., 218-223.

Liu, S., Q. Xu, and P. Zhang, 2005: Quality control of Doppler velocities contaminated by migrating birds. Part II: Bayes identification and probability tests. J. Atmos. Oceanic Technol., 22, 1114-1121, https://doi.org/10.1175/JTECH1758.1.

_- , and Coauthors, 2016: WSR-88D radar data processing at NCEP. Wea. Forecasting, 31, 2047-2055, https://doi.org/ 10.1175/WAF-D-16-0003.1.

Melnikov, V. M., R. J. Doviak, D. S. Zrnić, and D. J. Stensrud, 2011: Mapping Bragg scatter with a polarimetric WSR-88D. J. Atmos. Oceanic Technol., 28, 1273-1285, https://doi.org/ 10.1175/JTECH-D-10-05048.1.

,,$-- \ldots$, and,- 2013 : Structures of Bragg scatter observed with the polarimetric WSR-88D. J. Atmos. Oceanic Technol., 30, 1253-1258, https://doi.org/10.1175/JTECH-D-12-00210.1.

Rabin, R., and R. J. Doviak, 1989: Meteorological and astronomical influences on radar reflectivity in the convective boundary layer. J. Appl. Meteor., 28, 1226-1235, https://doi.org/10.1175/ 1520-0450(1989)028<1226:MAAIOR > 2.0.CO;2.

Richardson, L. M., J. G. Cunningham, W. D. Zittel, R. R. Lee, R. L. Ice, V. M. Melnikov, N. P. Hoban, and J. G. Gebauer, 2017: Bragg scatter detection by the WSR-88D. Part I: Algorithm development. J. Atmos. Oceanic Technol., 34, 465-478, https:// doi.org/10.1175/JTECH-D-16-0030.1.

Simmons, K., and D. Sutter, 2005: WSR-88D radar, tornado warnings, and tornado casualties. Wea. Forecasting, 20, 301310, https://doi.org/10.1175/WAF857.1.

Stumpf, G., A. Witt, E. D. Mitchell, P. L. Spencer, J. T. Johnson, M. D. Eilts, K. W. Thomas, and D. W. Burgess, 1998: The
National Severe Storms Laboratory mesocyclone detection algorithm for the WSR-88D. Wea. Forecasting, 13, 304-326, https://doi.org/10.1175/1520-0434(1998)013<0304:TNSSLM> 2.0.CO;2.

Whiton, R. C., P. L. Smith, S. G. Bigler, K. E. Wilk, and A. C. Harbuck, 1998: History of operational use of weather radar by U.S. weather services. Part II: Development of operational Doppler weather radars. Wea. Forecasting, 13, 244 252, https://doi.org/10.1175/1520-0434(1998)013<0244: HOOUOW $>2.0 . \mathrm{CO} ; 2$.

Witt, A., R. A. Brown, and Z. Jing, 2009: Performance of a new velocity dealiasing algorithm for the WSR-88D. Preprints, 34rd Conf. on Radar Meteorology, Williamsburg, VA, Amer. Meteor. Soc., P4.8, https://ams.confex.com/ams/34Radar/ techprogram/paper_155951.htm.

Xu, Q., 2009: Bayesian perspective of the unconventional approach for assimilating aliased radar radial velocities. Tellus, $\mathbf{6 1 A}$, 631-634, https://doi.org/10.1111/j.1600-0870.2009.00413.x.

__ and K. Nai, 2012: An adaptive dealiasing method based on variational analysis for radar radial velocities scanned with small Nyquist velocities. J. Atmos. Oceanic Technol., 29, 17231729, https://doi.org/10.1175/JTECH-D-12-00145.1.

_ and - 2013: A two-step variational method for analyzing severely aliased radar velocity observations with small Nyquist velocities. Quart. J. Roy. Meteor. Soc., 139, 1904-1911, https:// doi.org/10.1002/qj.2075.

, L. Wei, and Q. Zhao, 2009: An unconventional approach for assimilating aliased radar radial velocities. Tellus, 61A, 621630, https://doi.org/10.1111/j.1600-0870.2009.00412.x. , P. Zhang, S. Liu, and D. Parrish, 2011: A VADbased dealiasing method for radar velocity data quality control. J. Atmos. Oceanic Technol., 28, 50-62, https://doi.org/ 10.1175/2010JTECHA1444.1.

- - - S. Liu, C. Karstens, T. Smith, and Q. Zhao, 2013: Improved Doppler velocity dealiasing for radar data assimilation and storm-scale vortex detection. Adv. Meteor., 2013, 562386, https://doi.org/10.1155/2013/562386.

Zhang, P., S. Liu, and Q. Xu, 2005: Quality control of Doppler velocities contaminated by migrating birds. Part I: Feature extraction and quality control parameters. J. Atmos. Oceanic Technol., 22, 1105-1113, https://doi.org/10.1175/JTECH1757.1.

Zittel, W. D., D. Saxion, R. Rhoton, and D. C. Crauder, 2008: Combined WSR-88D technique to reduce range aliasing using phase coding and multiple Doppler scans. 24th Conf. on Integrated Information and Processing, New Orleans, LA, Amer. Meteor. Soc., P2.9, https://ams.confex.com/ams/88Annual/ techprogram/paper_131757.htm. 